\title{
Freeze-in dark matter production: models and phenomenology
}

\section{Andreas Goudelis*}

Sorbonne Université, CNRS, Laboratoire de Physique Théorique et Hautes Énergies, LPTHE, F-75252 Paris, France and Sorbonne Universités, Institut Lagrange de Paris (ILP), 98 bis Boulevard Arago, 75014 Paris, France

E-mail: andreas.goudelisalpthe.jussieu.fr

\begin{abstract}
We discuss theoretical and phenomenological aspects of the freeze-in cosmological dark matter production mechanism. After a short description of the main ideas behind freeze-in, we present a class of models which can naturally accommodate the extremely small dark matter couplings required for successful freeze-in, by relying on the Clockwork mechanism. Secondly, we discuss some phenomenological signatures that can arise in freeze-in models, in particular related to different searches for long-lived particles at the Large Hadron Collider.
\end{abstract}

Corfu Summer Institute 2018 "School and Workshops on Elementary Particle Physics and Gravity" (CORFU2018)

31 August - 28 September, 2018

Corfu, Greece

${ }^{*}$ Speaker. 


\section{Introduction}

The existence of dark matter is, today, hardly contested. Indeed, there exists currently no cosmological model which can simultaneously explain galactic rotation curves, gravitational lensing observations and, above all, the Cosmic Microwave Background (CMB) without invoking some amount of dark matter. Among these observations, the CMB further provides us with a valuable piece of information, namely the abundance of dark matter within $\Lambda \mathrm{CDM}$ cosmology [1]. Explaining why there is as much dark matter in the Universe as we infer from the CMB is one of the central questions in dark matter physics and, in some sense, one of the defining points of any dark matter model.

For several decades, the question of the cosmic dark matter abundance has been mostly addresed in the context of thermal freeze-out. However, the absence of conclusive signals in direct [2], indirect [3, 4] and collider [5, 6] searches (most of which were motivated exactly by this picture!) has put severe bounds on models of frozen-out dark matter. In this spirit, in recent years there has been an upsurge of interest in alternative dark matter generation mechanisms such as the so-called freeze-in mechanism [7, 8]. In the following, after swiftly introducing freeze-in we will briefly discuss aspects related to the theory and phenomenology of freeze-in models.

\section{Freeze-in: General framework}

Let us denote the dark matter particles by $\chi$, and let's consider all possible reactions $A \rightarrow B$ in which $\xi_{A}\left(\xi_{B}\right)$ particles of type $\chi$ are destroyed (created). The number density evolution of $\chi$ is described by a Boltzmann equation as

$$
\dot{n}_{\chi}+3 H n_{\chi}=\sum_{A, B}\left(\xi_{B}-\xi_{A}\right) \mathscr{N}(A \rightarrow B)
$$

where $H$ is the Hubble constant, $\mathscr{N}(A \rightarrow B)$ is the integrated collision term for the process $A \rightarrow B$ (describing the number of $A \rightarrow B$ reactions that take place per unit spacetime volume) and the sum runs over all possible reactions involving $\chi$ particles.

Freeze-in dark matter production relies on two basic premises. The first one is that dark matter (effectively) interacts extremely weakly (feebly) with the Standard Model sector as well as with all exotic particle species that are in chemical equilibrium with it. The second one is that at sufficiently early times, the dark matter abundance was negligible. Then, since the dark matter (self)annihilation rate scales as $n_{\chi}^{2}\langle\sigma v\rangle_{\chi+\chi \rightarrow \text { all }}$, we can ignore the dark matter depletion processes in Eq.(2.1) and only consider terms with $\xi_{A}=0$. In other words, in the freeze-in picture dark matter is only produced through annihilations of Standard Model (or other bath) particles or from decays of heavier, potentially exotic ones, until e.g. its production becomes either kinematically disfavoured or until the parent particles disappear from the plasma. Throughout the following, all dark matter abundance numerical computations that will be presented make use of micrOMEGAs 5 [10].

One of the attractive features of conventional thermal freeze-out has traditionally been the so-called "WIMP miracle", i.e. the fact that for a typical electroweak scale dark matter mass and interaction strength with the Standard Model particles, the dark matter abundance as measured through the CMB can be typically recovered. This feature created the hope that perhaps the dark 
matter question might be possible to address simultaneously with additional shortcomings of the Standard Model, notably the problem of the radiative stabilisation of the Higgs boson mass which implied the existence of New Physics not far from the electroweak scale. In the case of freeze-in, such a coincidence is harder to find. Indeed, if we assume that dark matter is produced from the annihilation of two Standard Model particles through a $Z^{\prime}$ gauge boson with $m_{Z^{\prime}} \sim \mathscr{O}\left(10^{2}\right) \mathrm{GeV}$, the product of the $Z^{\prime}$ couplings to dark matter and Standard Model particle pairs has to be of the order of $10^{-11}$. Similarly, if dark matter is produced from the decays of a heavier parent particle, the relevant coupling needs to be of the order of $10^{-13}$ [8]. Such small numbers may appear to be hard to justify from a theoretical standpoint. At the same time, if indeed dark matter consists of such Feebly Interacting Massive Particles (FIMPs) it becomes an issue whether the freeze-in mechanism can give rise to any observable signals at all. The first of these questions will be discussed in Section 3, whereas the second one in Section 4.

\section{Clockwork FIMPs}

As we mentioned, freeze-in involves feeble couplings between dark matter and the Standard Model. Whereas from a phenomenological standpoint such couplings are perfectly acceptable (or even appealing, cf Section 4), from a theoretical standpoint they might appear to be puzzling. Indeed, one would expect that in a natural theory all dimensionless couplings should be of $\mathscr{O}(1)$ or, at least, possible to relate to $\mathscr{O}(1)$ couplings in a dynamical manner.

So far, two main avenues have been explored in order to naturally generate the small couplings required for successful freeze-in. The first relies on scale suppression. Put simply, if e.g. dark matter is produced upon annihilation of Standard Model particles through a heavy mediator with a mass far above the reheating temperature, the effective low-energy interaction which is responsible for dark matter production will be suppressed, even though from a UV standpoint the mediator may interact quite strongly with the other particles in the spectrum [9]. A second way to suppress the strength of the interaction between dark matter and the visible sector is to rely on symmetries and symmetry breaking patterns. Such a possibility was studied in [11] and then [12, 13], focusing on the so-called "Clockwork" mechanism [14, 15, 16].

In [13] we studied two "Clockwork FIMP" scenarios, for scalar and fermionic dark matter. In the scalar case, we introduce a global $\prod_{i=0}^{N} U(1)_{i}$ symmetry group (the "Clockwork chain") which is spontaneously broken at some scale $f$, resulting in $N+1$ massless scalars at energies below $f$. Note that the various $U(1)_{i}$ factors may also be broken at different scales $f_{i}$. We take a single scale for simplicity. In addition to this spontaneous breaking, we further introduce an explicit soft breaking through $N$ spurion-like mass parameters $m_{j}^{2}$ charged as

$$
Q_{i}=\delta_{i j}-q \delta_{i(j+1)}
$$

under $U(1)_{i}$. For simplicity we again assume all $m_{j}^{2}$ parameters to be equal to a common value $m^{2}$. Since only $N$ out of the $N+1 U(1)$ factors are explicitly broken in this manner, there remains one massless Goldstone scalar (the "zero mode" $a_{0}$ ), whereas all other states in the Clockwork chain (the "Clockwork gears" $a_{k}$ ) acquire masses, for details $c f$ [13]. The zero mode will be our dark matter candidate. Next, we couple the $N$-th site of the Clockwork chain to the Standard Model through a Higgs portal term $\kappa\left|H^{\dagger} H\right| \phi_{N}^{2}$ and set $\kappa$ to its maximally natural value of 1 . The crucial 
observation is that the Clockwork zero mode only interacts with the Higgs doublet (as well as with the Clockwork gears) through terms scaling as inverse powers of $q^{N}$, hence, for large enough $q$ and $N$ these interactions can be suppressed. Of course, dark matter cannot be massless. In order to give it an adequate mass, we introduce an additional mass term for all sites of the quiver by adding a set of terms as $\sum_{i=0}^{N}\left(t^{2} / 2\right) \phi_{i}^{2}$. The full Lagrangian for our scalar FIMP model is, then, given by

$$
\mathscr{L}_{\text {sFIMP }}=\mathscr{L}_{\text {kin }}-\frac{1}{2} \sum_{i, j=0}^{N} \phi_{i} M_{i j}^{2} \phi^{j}-\frac{m^{2}}{24 f^{2}} \sum_{i, j=0}^{N}\left(\phi_{i} \tilde{M}_{i j}^{2} \phi^{j}\right)^{2}-\kappa\left|H^{\dagger} H\right| \phi_{n}^{2}+\sum_{i=0}^{n} \frac{t^{2}}{2} \phi_{i}^{2}
$$

Upon diagonalisation of the scalar mass matrix, we indeed obtain a FIMP zero mode with a mass controlled by the $t$ parameter (there is also an additional contribution coming from the Higgs portal term but for large enough $(q, N)$ values this is extremely suppressed) and its couplings to the Standard Model (and the Clockwork gears) suppressed by powers of $q^{N}$. Note that the Clockwork gears are not FIMPs and they thermalise with the Standard Model in the early Universe.

A fermion Clockwork FIMP can be constructed in a similar manner, but in this case it is chiral symmetry that protects the zero mode. We introduce $N+1$ right-handed chiral fermions $\psi_{R, j}, j=0, \ldots, N$ and $N$ left-handed chiral fermions $\psi_{L, i}, i=0, \ldots, N-1$. The chiral symmetry is broken by $N$ mass parameters $m_{i}$, which we again take to be equal to a common value $m$, as well as $N$ linking mass parameters $m q_{i}$ that induce nearest-neighbour interactions. Again, since only $N$ out of the $N+1$ sites are broken by the mass parameters, a remnant right-handed chiral fermion remains massless. In order to write down interactions between the Clockwork chain and the Standard Model we further introduce a set of vector-like leptons $L^{\prime}=\left(l_{1}, l_{2}\right)$ and $R^{\prime}=\left(r_{1}, r_{2}\right)$ transforming as $(\mathbf{1}, \mathbf{2},-\mathbf{1} / \mathbf{2})$ under $S U(3)_{c} \times S U(2)_{L} \times U(1)_{Y}$ and impose a discrete $\mathscr{Z}_{2}$ symmetry under which the SM is even and all other particles are odd. The last site of the Clockwork chain $\left(\psi_{R, N}\right)$ is, then, coupled to the Standard Model Higgs through a Yukawa coupling involving the lefthanded exotic lepton doublet. Lastly, we also introduce Majorana mass terms (which also break the quiver) to every side for both the left- and right-handed fermions. All in all, our Lagrangian in this case reads

$$
\mathscr{L}_{f F I M P}=\mathscr{L}_{F C W}+i \bar{L}^{\prime} \not D L^{\prime}+i \bar{R}^{\prime} \not D R^{\prime}+M_{D}\left(\bar{L}^{\prime} R^{\prime}\right)+Y \overline{L^{\prime}} \tilde{H} \psi_{R, N}+\text { h.c }
$$

where

$$
\begin{aligned}
\mathscr{L}_{F C W} & =\mathscr{L}_{k i n}-m \sum_{i=0}^{N-1}\left(\bar{\psi}_{L, i} \psi_{R, i}-q \bar{\psi}_{L, i} \psi_{R, i+1}+h . c\right)-\frac{M_{L}}{2} \sum_{i=0}^{N-1}\left(\bar{\psi}_{L, i}^{c} \psi_{L, i}\right)-\frac{M_{R}}{2} \sum_{i=0}^{N}\left(\bar{\psi}_{R, i}^{c} \psi_{R, i}\right) \\
& =\mathscr{L}_{k i n}-\frac{1}{2}\left(\bar{\Psi}^{c} \mathscr{M} \Psi+\text { h.c }\right)
\end{aligned}
$$

is the original fermion Clockwork Lagrangian. Once again, diagonialisation of the fermion mass matrix amounts to a zero mode with suppressed interactions which can play the role of a FIMP dark matter candidate.

In summary, starting from a theory with $\mathscr{O}(1)$ couplings, for $q>1$ and a sufficiently large number of sites $N$ the interactions between the zero mode and the Standard Model (as well as all particles that are in thermal equilibrium with the latter) can become extremely suppressed and we can obtain a viable FIMP dark matter candidate. This is illustrated in figure 1, in which we delineate 

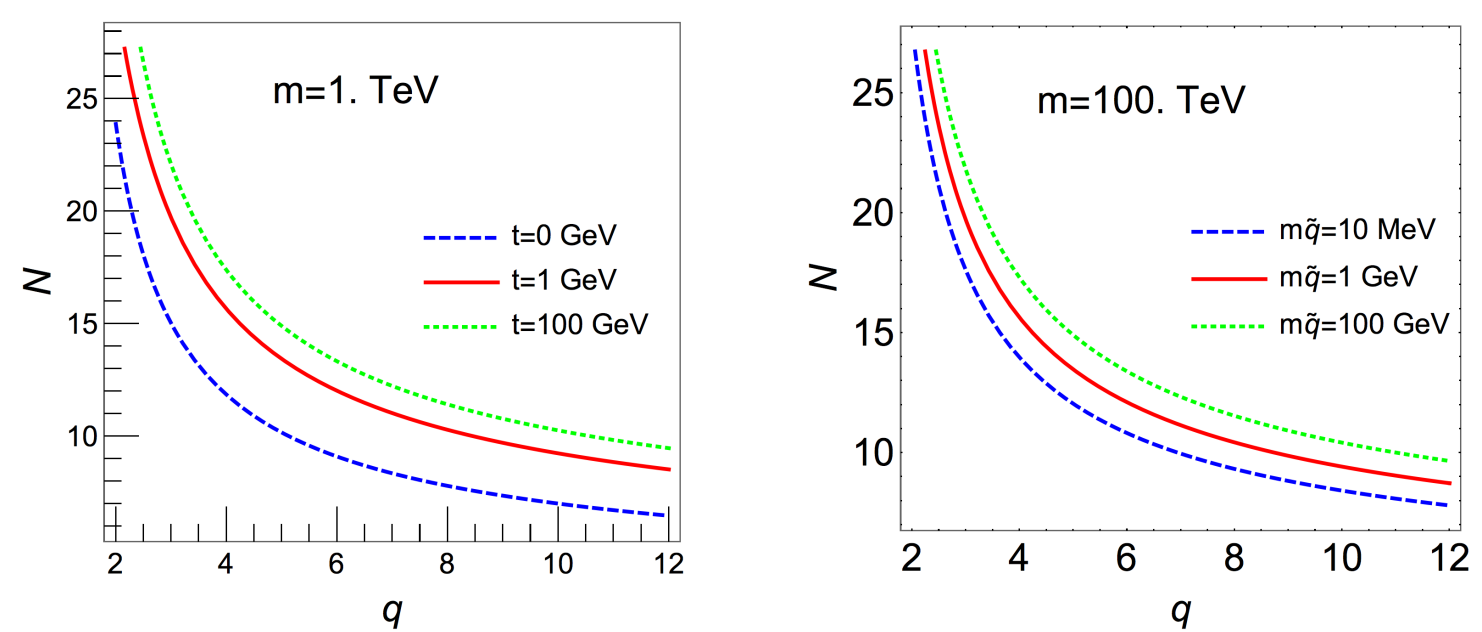

Figure 1: Contours indicating the values of $q$ and $N$ for which the observed dark matter abundance can be reproduced according to the freeze-in mechanism for our scalar (left) and fermion (right) models.

the $(q, N)$ combinations for which the frozen-in zero mode can account for the observed dark matter abundance in the Universe in our scalar (left panel) and our fermion models (right panel), for three discrete choices of the $t$ mass parameter and $m \tilde{q}$ respectively. Note that in both cases the dark matter is mainly populated due to the decays of the Clockwock gears, while contributions from scattering processes have been found to be subleading. Lastly, let us comment on the fact that alternative Clockwork-based constructions can also be envisaged, for examples and a relevant discussion $c f$ the original reference [13].

\section{Collider signatures of freeze-in dark matter}

Having presented a mechanism in order to naturally generate the small couplings that are necessary for successful freeze-in, let us now move to the phenomenology of freeze-in models. As we already alluded to, since in freeze-in dark matter is required to be (at least effectively) a FIMP, it is expected to neither annihilate substantially into Standard Model particles (this is one of the requirements of freeze-in, after all) nor to scatter strongly off ordinary matter (although for an exception $c f$ e.g. [17]). Similarly, direct FIMP pair-production at high-energy colliders is expected to occur at an unobservable rate. However, in freeze-in models not all exotic particles need to be feebly coupled (we actually just saw two examples in Section 3). Then if, for instance, there exists some heavier particle which possesses substantial interactions with the Standard Model and which has to, eventually, decay into dark matter, we could hope for such particles to be copiously produced e.g. at the LHC. In [18] we studied a class of models offering such a possibility.

Let us consider an extension of the SM by a real scalar DM candidate $s$ that is neutral under $S U(3)_{c} \times S U(2)_{L} \times U(1)_{Y}$, along with a vector-like fermion $F$. In all cases we take $F$ to be an $S U$ (2) singlet (for an alternative possibility $c f$ e.g. [19]). Both $s$ and $F$ are taken to be odd under a $\mathbf{Z}_{2}$ symmetry which, by choosing $m_{s}<m_{F}$, stabilizes the DM candidate. Lastly, the SM particles are taken to transform trivially under the same discrete symmetry. 
In such a scenario the dark matter couples to the Standard Model through Yukawa-type interactions involving the left-handed component of the vector-like fermion and the Standard Model right-handed fermions: up-type quarks, down-type quarks and charged leptons. In all three cases, the Lagrangian can be written as

$$
\begin{aligned}
\mathscr{L} & =\mathscr{L}_{\mathrm{SM}}+\partial_{\mu} s \partial^{\mu} s-\frac{\mu_{s}^{2}}{2} s^{2}+\frac{\lambda_{s}}{4} s^{4}+\lambda_{s h} s^{2}\left(H^{\dagger} H\right) \\
& +\bar{F}(i \not D) F-m_{F} \bar{F} F-\sum_{f} y_{s}^{f}\left(s \bar{F}\left(\frac{1+\gamma^{5}}{2}\right) f+\text { h.c. }\right),
\end{aligned}
$$

where $f=\{e, \mu, \tau\},\{u, c, t\}$ or $\{d, s, b\}$, depending on the $S U(3)_{c} \times U(1)_{Y}$ transformation properties of $F$. For simplicity we focus on the possibilities $f=\{e, \mu\}$ and $f=\{u, c\}$.

In these models dark matter can, indeed, be cosmologically produced from the decays of the heavier vector-like fermions which can carry $U(1)_{Y}$, or even colour charge. One also finds that when such decays dominate dark matter production, the parent particle decay length can be approximated as:

$$
c \tau \approx 4.5 \mathrm{~m} \xi g_{F}\left(\frac{0.12}{\Omega_{s} h^{2}}\right)\left(\frac{m_{s}}{100 \mathrm{keV}}\right)\left(\frac{200 \mathrm{GeV}}{m_{F}}\right)^{2}\left(\frac{102}{g_{*}\left(m_{F} / 3\right)}\right)^{3 / 2}\left[\frac{\int_{m_{F} / T_{R}}^{m_{F} / T_{0}} d x x^{3} K_{1}(x)}{3 \pi / 2}\right] .
$$

From this relation we deduce that for a fixed parent particle mass, the decay length required in order to reproduce the observed dark matter abundance is of a macroscopic size, unless dark matter is extremely light (but one obtains a bound $m_{s}>12 \mathrm{keV}$ from Lyman- $\alpha$ forest observations) or if the reheating temperature $T_{R}$ is very low (keeping in mind that it must be higher than the temperatures of Big Bang Nucleosynthesis). We see, then, that freeze-in can naturally drive us into the realm of searches for long-lived particles at the LHC.

We consider three types of searches: if the parent particle is sufficiently short-lived, its visible decay products can be reconstructed as displaced leptons (if $F$ is a "heavy lepton") or jets (if $F$ is a "heavy quark"). If, on the other hand the parent particle lifetime is long enough, then it will traverse the LHC detectors and behave as a Heavy Stable Charged Particle (HSCP), leaving behind anomalous tracks, energy depositions and, potentially signals in the muon chambers. As we will see shortly, these two types of searches turn out to be sufficient to cover an important part of the parameter space of our heavy quark model. In the case of our leptonic model, sensitivity is lost for intermediate lifetimes $(c \tau \sim 1 \mathrm{~m})$. In this region of parameter space, it is possible that the parent particle may decay within the tracker, with its track being possible to reconstruct, but with the outgoing Standard Model lepton track failing reconstruction. We can, then, obtain a "disappearing track" signature, which is currently being looked for by both ATLAS and CMS. In our analysis we have used searches for displaced leptons (vertices) in CMS (ATLAS), for disappearing tracks in both experiments and for HSCPs in CMS. A detailed description of the analyses, the relevant references and the recasting procedure can be found in [18].

Our results are presented in figure 2 for the leptonic (upper panel) and quark model (lower panel), where we show the $\left(m_{F}, c \tau\right)$ combinations which are excluded by the different LHC searches as explained in the legend. Along the black lines, the dark matter density constraint can be satisfied assuming different values for the dark matter mass and the reheating temperature. 

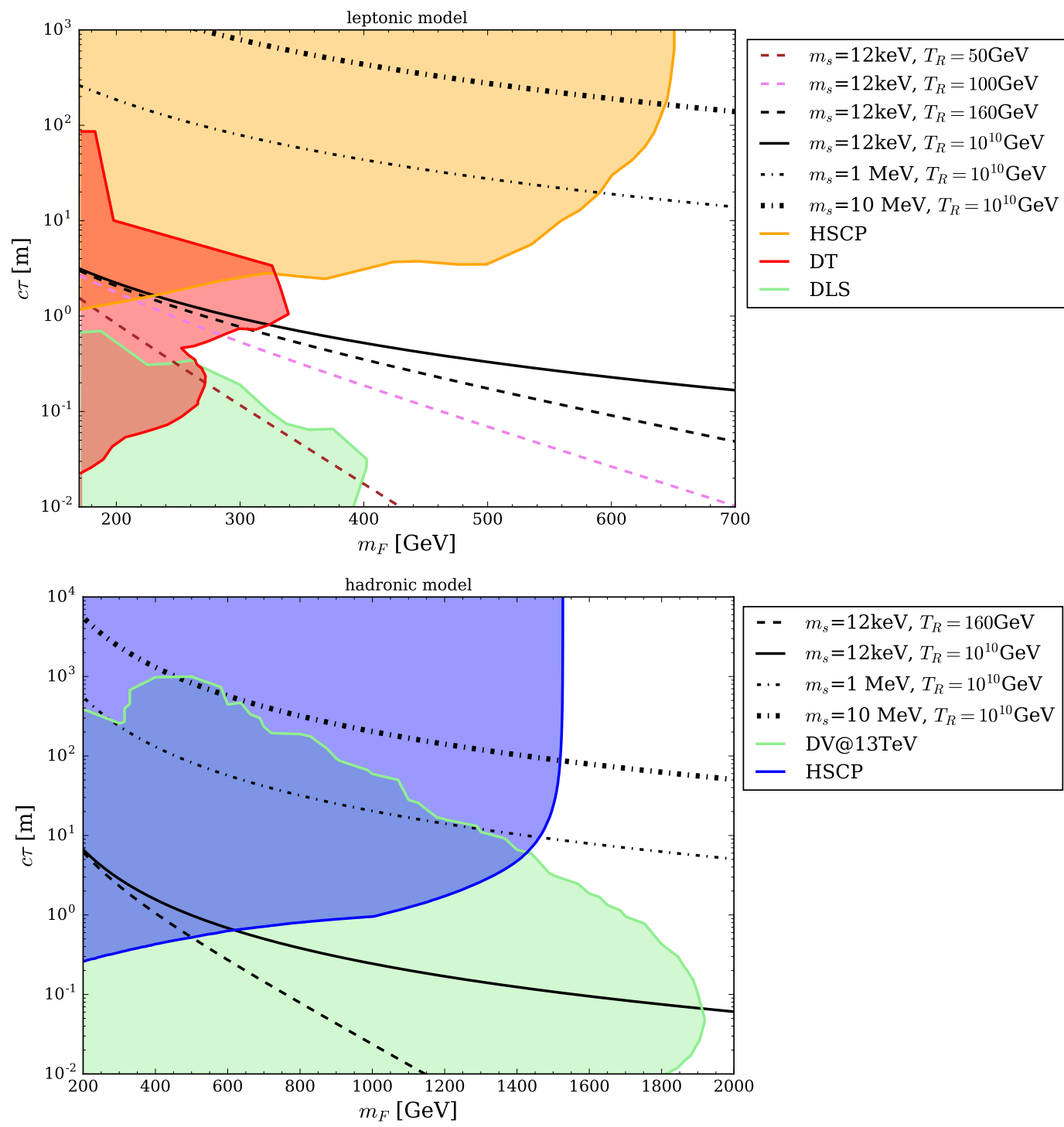

- - $m_{s}=12 \mathrm{keV}, T_{R}=160 \mathrm{GeV}$

- $m_{s}=12 \mathrm{keV}, T_{R}=10^{10} \mathrm{GeV}$

․ $m_{s}=1 \mathrm{MeV}, T_{R}=10^{10} \mathrm{GeV}$

$\because m_{s}=10 \mathrm{MeV}, T_{R}=10^{10} \mathrm{GeV}$ - DV@13TeV

- HSCP

Figure 2: Constraints from different LHC searches for long-lived particles on the $\left(m_{F}, c \tau\right)$ plane for our leptonic (upper panel) and hadronic (lower panel) model. Along the black lines the dark matter abundance as measured by the Planck mission is satisfied for different values of the dark matter mass and reheating temperature.

We observe that indeed, searches for long-lived particles can be powerful probes of freeze-in within the class of models we considered. They are already excluding parent particle masses up to several hundreds of $\mathrm{GeV}$ (lepton model) or even within the $\mathrm{TeV}$ region (quark model). Equally interestingly, we see clearly that different types of long-lived particle searches probe different regions of parameter space. In this sense, these searches are not only complementary with cosmological probes of dark matter but also with each other.

It should also be noted that a positive observation of displaced leptons could offer the possibility to infer information on the reheating temperature and, hence, put bounds on electroweak 
baryogenesis. For a more detailed exposition, we again refer the reader to the original reference [18].

\section{Summary and conclusions}

In summary, freeze-in is a viable dark matter generation mechanism in which feebly interacting dark matter particles are produced from annihilations or decays of other, more strongly coupled ones ${ }^{1}$. It can be accommodated within simple or more sophisticated extensions of the Standard Model, in which it is also possible to explain why dark matter would interact as weakly with the visible sector as required to satisfy the dark matter abundance constraint. Lastly, it can provide exciting phenomenological signatures, radically different with respect to those appearing in conventional thermal freeze-out models. Notably, freeze-in phenomenology is related to the flourishing activity of searches for long-lived particles at the Large Hadron Collider. Thankfully, in yet another case, dark matter physics can motivate an experimental search program at the Large Hadron Collider.

\section{Acknowledgments}

A.G. was supported by the Labex ILP (reference ANR-10-LABX-63) part of the Idex SUPER, and received financial state aid managed by the Agence Nationale de la Recherche, as part of the programme "Investissements d'Avenir" under the reference ANR-11-IDEX-0004-02. A.G. would like to thank the organisers of the organisers of the 2018 Corfu Workshop on the Standard Model and Beyond for warm hospitality.

\section{References}

[1] P. A. R. Ade et al. [Planck Collaboration], Astron. Astrophys. 594, A13 (2016) doi:10.1051/0004-6361/201525830 [arXiv:1502.01589 [astro-ph.CO]].

[2] E. Aprile et al. [XENON Collaboration], Phys. Rev. Lett. 121, no. 11, 111302 (2018) doi:10.1103/PhysRevLett.121.111302 [arXiv:1805.12562 [astro-ph.CO]].

[3] M. L. Ahnen et al. [MAGIC and Fermi-LAT Collaborations], JCAP 1602, no. 02, 039 (2016) doi:10.1088/1475-7516/2016/02/039 [arXiv:1601.06590 [astro-ph.HE]].

[4] H. Abdallah et al. [H.E.S.S. Collaboration], Phys. Rev. Lett. 117, no. 11, 111301 (2016) doi:10.1103/PhysRevLett.117.111301 [arXiv:1607.08142 [astro-ph.HE]].

[5] A. M. Sirunyan et al. [CMS Collaboration], Phys. Rev. D 97, no. 9, 092005 (2018) doi:10.1103/PhysRevD.97.092005 [arXiv:1712.02345 [hep-ex]].

[6] M. Aaboud et al. [ATLAS Collaboration], JHEP 1801, 126 (2018) doi:10.1007/JHEP01(2018)126 [arXiv:1711.03301 [hep-ex]].

[7] J. McDonald, Phys. Rev. Lett. 88, 091304 (2002) doi:10.1103/PhysRevLett.88.091304 [hep-ph/0106249].

\footnotetext{
${ }^{1}$ It is also possible that FIMP dark matter could be produced from the decays of another frozen-in FIMP. This possibility of "freeze-in from freeze-in" goes beyond the scope of this short presentation.
} 
[8] L. J. Hall, K. Jedamzik, J. March-Russell and S. M. West, JHEP 1003, 080 (2010) doi:10.1007/JHEP03(2010)080 [arXiv:0911.1120 [hep-ph]].

[9] Y. Mambrini, K. A. Olive, J. Quevillon and B. Zaldivar, Phys. Rev. Lett. 110, no. 24, 241306 (2013) doi:10.1103/PhysRevLett.110.241306 [arXiv:1302.4438 [hep-ph]].

[10] G. Bélanger, F. Boudjema, A. Goudelis, A. Pukhov and B. Zaldivar, Comput. Phys. Commun. 231, 173 (2018) doi:10.1016/j.cpc.2018.04.027 [arXiv:1801.03509 [hep-ph]].

[11] J. Kim and J. McDonald, Phys. Rev. D 98, no. 2, 023533 (2018) doi:10.1103/PhysRevD.98.023533 [arXiv:1709.04105 [hep-ph]].

[12] J. Kim and J. Mcdonald, Phys. Rev. D 98, no. 12, 123503 (2018) doi:10.1103/PhysRevD.98.123503 [arXiv:1804.02661 [hep-ph]].

[13] A. Goudelis, K. A. Mohan and D. Sengupta, JHEP 1810, 014 (2018) doi:10.1007/JHEP10(2018)014 [arXiv:1807.06642 [hep-ph]].

[14] D. E. Kaplan and R. Rattazzi, Phys. Rev. D 93, no. 8, 085007 (2016) doi:10.1103/PhysRevD.93.085007 [arXiv:1511.01827 [hep-ph]].

[15] K. Choi and S. H. Im, JHEP 1601, 149 (2016) doi:10.1007/JHEP01(2016)149 [arXiv:1511.00132 [hep-ph]].

[16] G. F. Giudice and M. McCullough, JHEP 1702, 036 (2017) doi:10.1007/JHEP02(2017)036 [arXiv:1610.07962 [hep-ph]].

[17] T. Hambye, M. H. G. Tytgat, J. Vandecasteele and L. Vanderheyden, Phys. Rev. D 98, no. 7, 075017 (2018) doi:10.1103/PhysRevD.98.075017 [arXiv:1807.05022 [hep-ph]].

[18] G. Bélanger et al., JHEP 1902, 186 (2019) doi:10.1007/JHEP02(2019)186 [arXiv:1811.05478 [hep-ph]].

[19] L. Calibbi, L. Lopez-Honorez, S. Lowette and A. Mariotti, JHEP 1809, 037 (2018) doi:10.1007/JHEP09(2018)037 [arXiv:1805.04423 [hep-ph]]. 